\title{
HEAT AND MASS TRANSFER IN THERMAL PLASMAS
}

\author{
I. G. SAYCE \\ Division of Chemical Standards, National Physical Laboratory, Teddington, Middlesex, TW11 0LW, UK
}

\begin{abstract}
The present status of plasma processes involving heat and mass transfer is surveyed, with particular reference to the processes of vaporization, condensation and chemical reaction. Some of the more important plasma techniques are discussed with emphasis placed on means for maximising the overall thermal efficiency of the mass transfer process, and for controlling the properties of the condensed phase produced. Aspects of current theories concerning the above processes are also discussed.
\end{abstract}

\section{INTRODUCTION}

Whenever a condensed phase exists in contact with a thermal plasma there occur the simultaneous processes of heat and mass transfer. Even when no vaporization or chemical reaction of the condensed phase takes place, there remains an important element of mass transfer, in that the process of convective heat transfer occurs in part by the recombination of dissociated species at the interface, and transport of species to and from the interface is thus an essential part of the process. To do justice to both practical and theoretical aspects of this broad subject would be impossible in a single paper, and a somewhat selective approach will therefore be adopted.

The processes which will be discussed are those of vaporization, and the closely related subject of chemical reaction when this involves reaction with (or evolution of) gas, and condensation from the vapour phase. All have been extensively studied with varying degrees of success, and all have important commercial applications. Since it appears unsatisfactory to attempt to separate the theory of these processes from their practice, the treatment adopted will place some emphasis on the practical implications of the subject, in an endeavour to point the way toward the most efficient means of perfecting the process under consideration. Again, since experimental practice almost invariably leads and theoretical understanding follows, the practical aspects will be considered first.

\section{VAPORIZATION AND CHEMICAL REACTION}

The process of vaporization is perhaps the most extensively studied of the areas selected, although it is not the most commercially important at this time. Arising perhaps from an early notion that the vaporization of metallic oxides might lead to new routes to the metals themselves, many metal oxides have been heated in, or passed through, thermal plasmas with a view to affecting vaporization or reduction. ${ }^{1}$ More recently it has been appreciated that while total vaporization might be an energetically inefficient route to most metals, it might well yield a viable process for preparing certain fine powders, and likewise offer a means of separating sufficiently valuable components from otherwise intractable mixtures. ${ }^{2}$ Many of the concepts developed in both practical and theoretical studies of vaporization have application also in the field of chemical reaction.

Early attempts at vaporization generally employed either the simple d.c. plasma jet, or induction plasma. The reason for this choicelay in the ready availability of the two types of device, rather than in their intrinsic efficiency for the purpose, which is in neither case high. Indeed of the many attempts to treat, i.e. vaporize or chemically react, powders in the efflux of a plasma jet or induction plasma, the results have generally been disappointing in terms of overall efficiency. It is useful to consider why.

Taking first the plasma jet, it is clearly desirable to inject the material to be treated into the hottest part of the plasma column. Electrode erosion and other design problems have generally prevented workers from feeding material through the current-carrying region of the arc, and therefore it has been necessary to inject material into the hot gas stream leaving the torch. This gives rise to several sources of inefficiency. Instead of using electrical energy to heat the powdered feedstock directly, i.e. to effect the desired process, the energy is first used to heat the gas, which can be done only at the intrinsic efficiency of the torch (typically 50-80\% in laboratory devices). Also, it is necessary to achieve sufficiently good mixing of feedstock with this hot gas, and to maintain an adequately long residence time, in order to attain the required thermal and chemical equilibrium.

Furthermore the entire process must be effected under conditions where heat losses to the surroundings are minimised. The high velocity, viscosity, and thermal gradients of the conventional plasma jet combine to ensure both that injection of particles into the hottest region of the gas is difficult, and their retention time is short. The entrainment of cold gas at the orifice of the torch further reduces the overall thermal efficiency.

Should vaporization begin to take place, heat transfer through the expanding envelope of relatively cool vapour products becomes progressively slower as the vaporization proceeds, particularly after the particles have achieved a velocity approaching that of the gas. For example, the total vaporization of a particle of silica of $200 \mu \mathrm{m}$ diameter at a temperature of $3000^{\circ} \mathrm{K}$ will lead to a vapour cloud of diameter $5 \mathrm{~mm}$ (assuming vaporization to form $\mathrm{SiO}+\frac{1}{2} \mathrm{O}_{2}$, and uniform temperature across the vapour cloud). Unless a condition of forced convection is achieved, complete vaporization of such particles requires heat transfer across a considerable distance from the perimeter of the vapour cloud to the centre. Such a process will only occur if there is available a substantial surplus of thermal energy, and a retention time far longer than the few milliseconds available in a conventional plasma jet. If it is required to vaporize not one but many such particles it is clear that some more efficient device must be found.

Similar arguments apply to the vaporization of particles in the induction plasma. Again the particles do not generally pass through the current-carrying region of the 
plasma, and difficulties are observed in projecting particles into the plasma fireball due to the countercurrent flows of hot gas which result from magneto-hydrodynamic effects within the plasma. ${ }^{3}$ Longer retention times are however observed when the particles are fed countercurrent-wise into the upward-flowing plasma, and this approach does provide a means of sweeping away the vapour cloud discussed above. ${ }^{4}$ The technique appears attractive for permitting the study and theoretical interpretation of heat transfer to individual particles, ${ }^{5}$ but in view of the basic inefficiency of the induction plasma it appears somewhat unlikely that this device will prove economic for particle vaporization on a commercial scale.

A number of schemes have been devised to increase both heat and mass transfer rates to above those possible in the preceding simple devices. One of the earliest, developed by Sheer and Korman, is the well-known high intensity arc. ${ }^{6}$ The material to be vaporized is generally compacted with carbon to form a consumable anode. As the arc current is raised, a critical current is reached when the anodic arc root extends over the face of the electrode, which is caused to evaporate rapidly to form a plume of vapour which streams away from the surface. Under these conditions the arc is said to operate in the high intensity mode, and a high proportion of the input energy dissipated within the arc is released in the anode fall zone adjacent to the electrode surface (Fig. 1). The use of the high intensity arc clearly obviates the need to employ an intermediate working gas, and the thermal efficiencies can be quite high $(9-18 \mathrm{kWh} / \mathrm{kg}$ for vaporization of silica at $7-9 \mathrm{~kg} / \mathrm{hr}$ at $70-150 \mathrm{~kW}$ input power $\left.{ }^{7}\right)$.

However one of the major disadvantages of this technique is the need to make strong, thermally shockresistant, electrodes. Another feature of the method is that all vapour products must be raised to a temperature of several thousand degrees in order to maintain the electrical conductivity of the arc. However silica (for example) may be vaporized at high rates in the presence of carbon at temperatures in the region of $2000^{\circ} \mathrm{C}$, and to
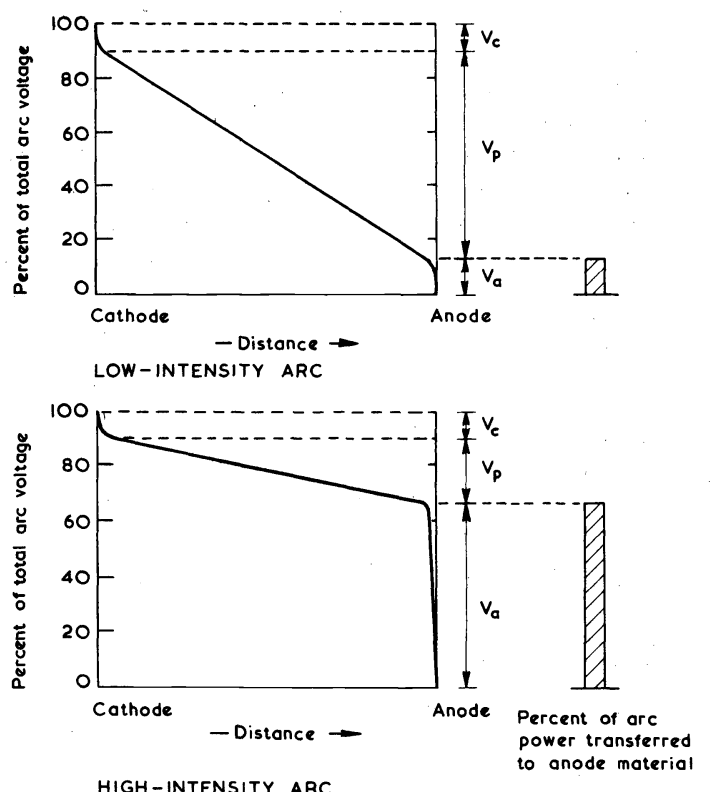

Fig. 1. Voltage distribution between electrodes in low and high intensity arcs: $V_{a}=$ voltage drop across anode sheath, $V_{c}=$ voltage drop across cathode sheath, $V_{p}=$ voltage drop across remainder of plasma column. heat the gases to higher temperature represents a waste of thermal energy.

A development of the concept of vaporization from the electrode surface, which also makes use of the heat in the arc tail-flame, has been used for the evaporation of silica. A d.c. plasma torch has been described which employs hollow cylindrical electrodes made of tungsten, ${ }^{8}$ Fig. 2. This was operated while pointing the reactor vertically downward and feeding a stream of silica carried in a gas vortex onto the inner wall of the lower electrode (usually cathode), or onto the wall of the tungsten tube which served to contain the tail-flame. The oxide melted on the reactor walls and flowed slowly downwards, surrounding the tail-flame. The cathodic arc root then moved freely over the surface of the molten silica, which received additional heat from the plasma column streaming through the reactor. The silica was thus vaporized continuously and with quite high thermal efficiency (24-53 kWh/kg), and on quenching the vapour from such a reactor a fine particle silica fume could be recovered. ${ }^{9}$ Relative to the conventional d.c. torch, this approach represents a useful means of simultaneously increasing the retention time and decreasing electrode heat losses. In this respect it has certain features in common with some forms of Gerdien torch. ${ }^{10}$

Another device which has been used to extend retention time and improve the thermal contact between the gas and the condensed phase is the centrifugal plasma furnace. This has now been used by several groups for melting and vaporizing refractory materials heated by the simple plasma torch, ${ }^{11-14}$ by a transferred arc between two plasma torches, ${ }^{15}$ and even by the induction plasma. ${ }^{16}$ The simple furnace, heated by a non-transferred plasma jet (Fig. 3) has been used to melt and vaporize such oxides as alumina, magnesia and silica, ${ }^{13}$ vaporization of which occurs via suboxide, or metallic species, and is thus greatly enhanced by the addition of gaseous or solid reducing agents. Thus in batch distillations of silica in the presence of carbon at $30 \mathrm{~kW}$, peak distillation rates of over $3 \mathrm{~kg} / \mathrm{hr}$ were observed, implying an energy requirement even in a small unoptimised furnace of under $10 \mathrm{kWh} / \mathrm{kg}$.

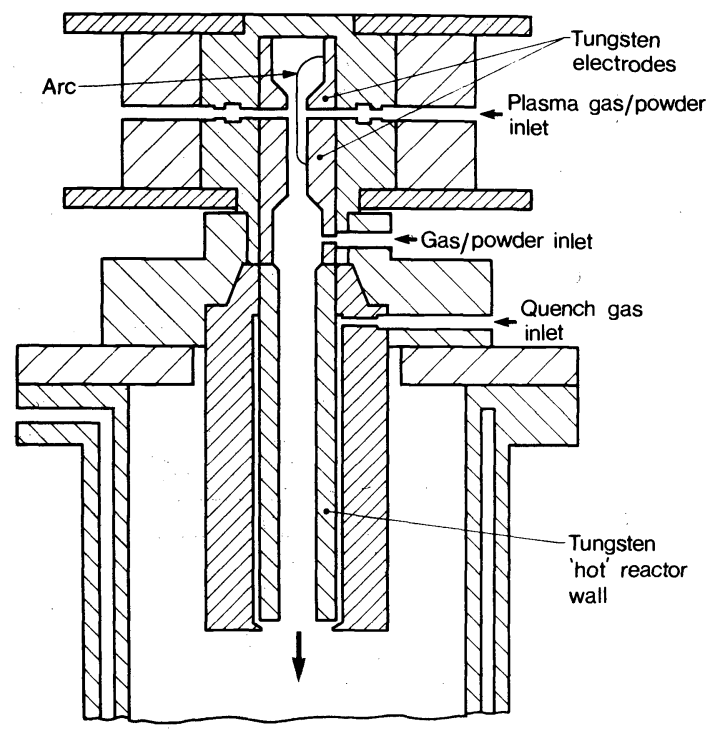

Fig. 2. Plasma reactor for silica vaporization, MHD Research Inc. ${ }^{8}$ 


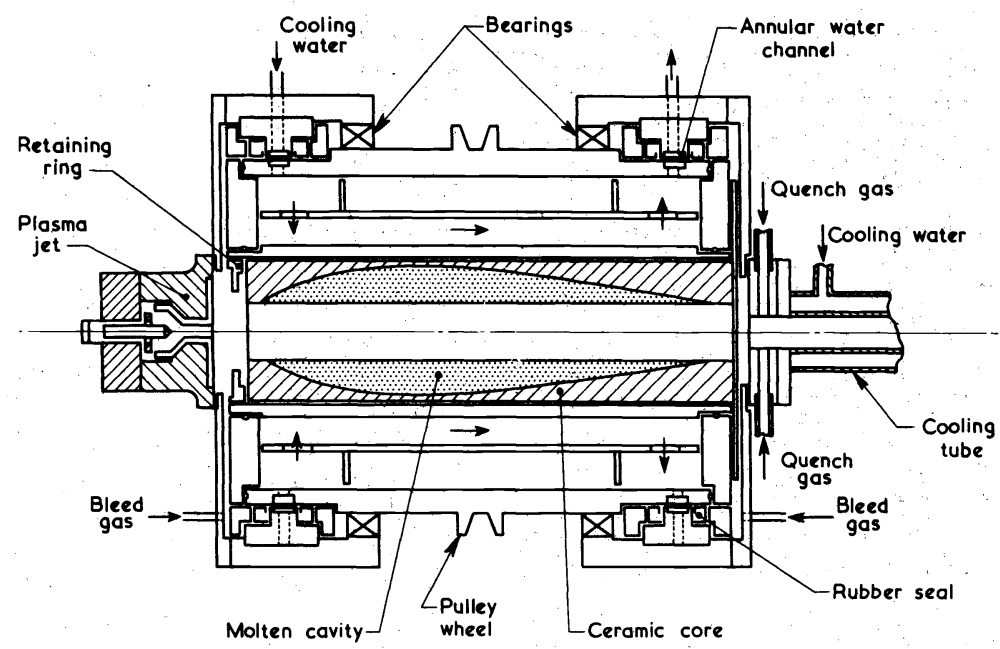

Fig. 3. Centrifugal plasma furnace heated by non-transferred DC plasma jet.

There are several sources of inefficiency in using the simple plasma jet to heat such a furnace. Firstly, the arc is again being used to heat the gas stream and a substantial proportion of the energy is lost to the torch cooling water. Secondly, it is necessary to transfer heat efficiently from the gas stream to the furnace contents in a separate stage. It would be preferable to associate these two processes as closely as possible. This has been achieved in a development of the centrifugal furnace in which an arc is struck between a consumable carbon electrode (or other form of cathode) and the melt itself, which acts as anode. ${ }^{17}$ The circuit is completed by means of a stationary brush mechanism at the end of the furnace (Fig. 4). The arc is struck initially to the carbon exit nozzle and the cathode is withdrawn through the furnace. Once the contents have melted, the liquid is sufficiently conducting to maintain the anode arc root, and the melt is thus heated by electron bombardment at this arc root, resistively by Joule heating, and also by conduction and radiation from the hot gas stream. The operation of the furnace is independent of any gas feed, and the concept appears to be one of the most efficient means available for evaporating molten ceramics.

Where the refractory can be made to sublime it may not be necessary to employ a rotating furnace, and in this case a stationary carbon arc furnace may well be adequate. ${ }^{18}$
Such a furnace has been used for the vaporization of silica in the presence of carbon, a relatively low temperature process, and a distillation rate of $54 \mathrm{~kg} / \mathrm{hr}$ at $500 \mathrm{~kW}$ (i.e. a power consumption of $9.3 \mathrm{kWh} / \mathrm{kg}$ ) has been reported.

Where it is possible to transfer the arc to the substrate to be vaporized, this represents the most efficient means of transferring electrically generated heat to that material. In some cases, however, this may be inconvenient, and in such circumstances transfer of the arc between two or more plasma torches represents a relatively efficient alternative; indeed 2-torch heating of both horizontal and the vertical centrifugal furnaces has been reported..$^{15,19}$ In the latter case there is evidence that some of the current flow may again be made to occur within the melt. The advantages of this approach over the non-transferred plasma jet are clear. The transferred arc is long and operates at relatively high voltage. Furthermore it may be effectively surrounded by the material to be treated, and is in proximity to water-cooled surfaces only at its extremities. Finally the thermal input may be made to be substantially independent of gas flow.

The vertical centrifugal plasma furnace (Fig. 5) has now been shown to be a thermally efficient tool for the continuous melting of ceramics. ${ }^{19}$ Thus at $60 \mathrm{~kW}$ mullite $\left(3 \mathrm{Al}_{2} \mathrm{O}_{3} .2 \mathrm{SiO}_{2}\right)$ has been melted at some $20 \mathrm{~kg} / \mathrm{hr}$, i.e. for a specific energy requirement of only $3 \mathrm{kWh} / \mathrm{kg}$. It has also

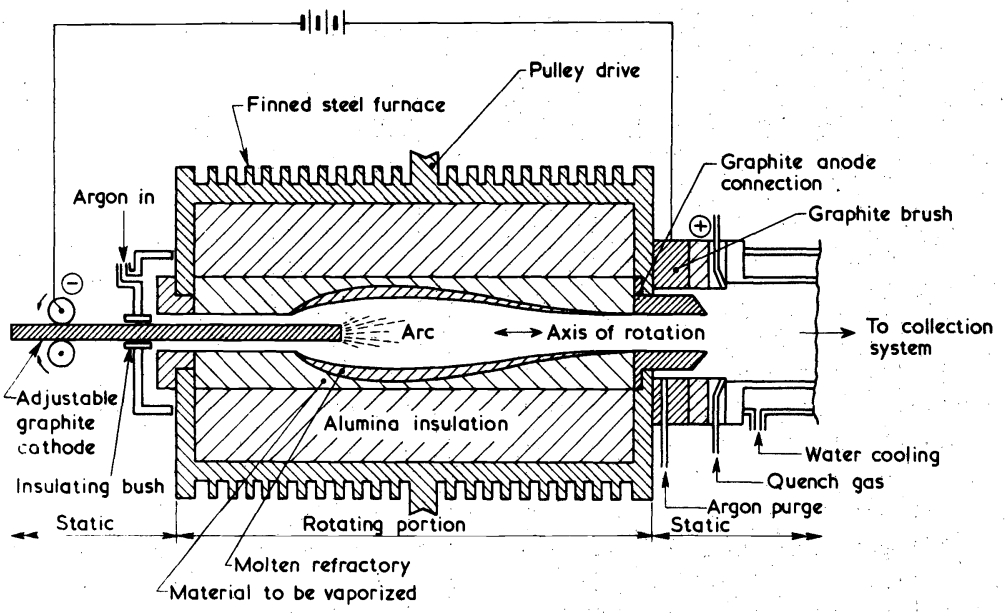

Fig. 4. Air-cooled centrifugal furnace heated by transferred DC arc. 


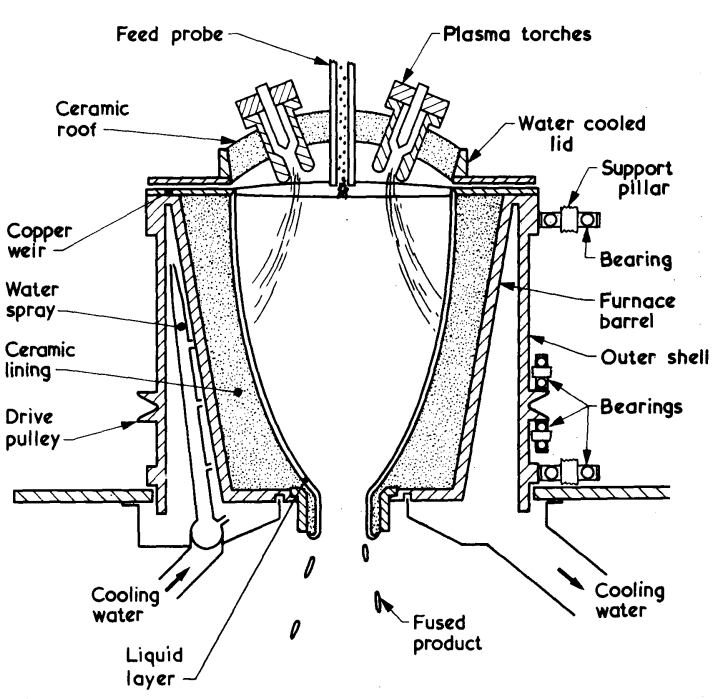

Fig. 5. Centrifugal furnace for continuous feed, heated by transferred arc between plasma jets.

yielded promising results for the continuous fuming of tin as oxide from low grade slags. ${ }^{2}$ Tin fuming is conventionally practised in the presence of sulphur, when the species $\mathrm{SnS}$ is volatilized. The method results in the evolution of large quantities of sulphur dioxide, but the resulting pollution can be avoided using the plasma furnace, by volatilisation of the lower oxide $\mathrm{SnO}$. Thus, operating at about $40 \mathrm{~kW}, 80 \%$ of the tin content has been removed from a $3.4 \%$ tin slag fed at $9 \mathrm{~kg} / \mathrm{hr}$, i.e. a slag treatment rate of $4.5 \mathrm{kWh} / \mathrm{kg}$. Lower specific energy requirements would of course be obtained if a sulphide fuming process were attempted. In related work involving arc transfer to tin silicate melts held in a conventional carbon arc furnace, oxide fuming has again been demonstrated. The use of such a transferred arc leads to efficient heat transfer and is also reported to give rise to an element of electrolysis in the fuming process. ${ }^{20,21}$

The above devices offer various means of vaporizing materials from the surface of the plasma-heated bulk melt. There are, however, situations where it is required to heat a particulate feedstock, where it may be required to effect vaporization from, or chemical reaction of, a stream of discrete particles. The difficulties of achieving this aim with the simple plasma heater have already been mentioned. It will be recalled that we require to bring the particles into close and prolonged thermal contact with the hot gas, if possible to introduce the particles into the current-carrying (energy dissipating) region of the plasma, and to arrange if possible to have the power input independent of gas flow, so that we are not heating an unnecessarily large volume of gas.

Early work on vaporization, using such an approach, was described by Whyman. ${ }^{22}$ A vertical d.c. arc was maintained between water-cooled electrodes, and this arc was surrounded by a water-cooled cylindrical rotor, Fig. 6 . The effect of this rotor was to establish a radial acceleration on the gas, superimposed upon the vertical gravitational field. Under these circumstances the convective cooling, which serves to constrict the normal vertical arc, is avoided, and the arc column expands outward to the walls of the rotor. Whyman et al. found that it was possible to feed particles into the arc column, by entrainment into the cathode jet flows which exist around any cathodic arc root, and to obtain vaporization of the particles so entrained. ${ }^{23}$

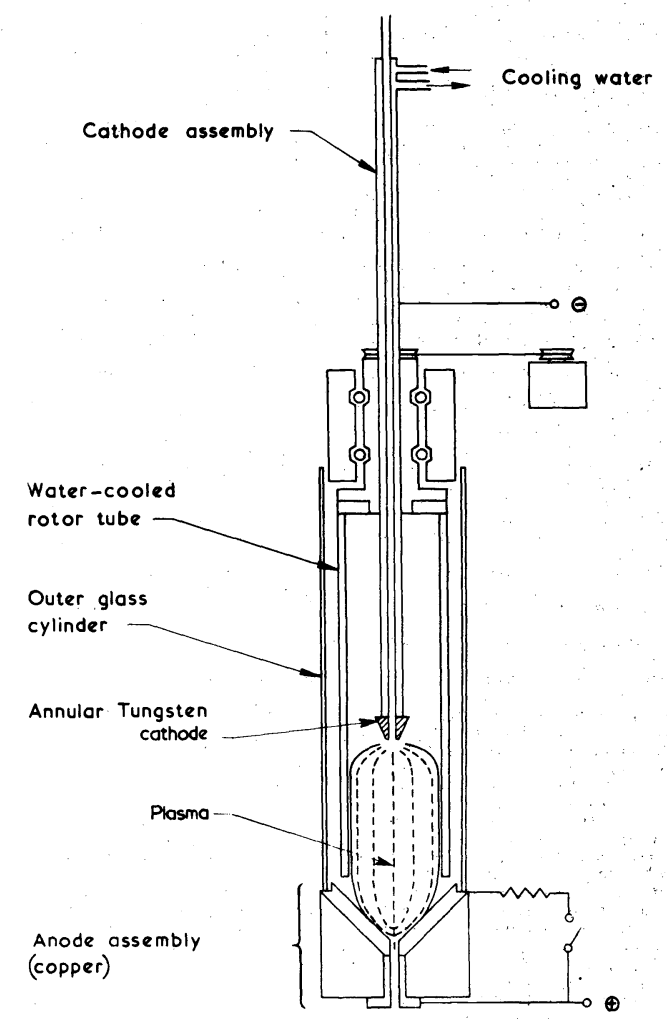

Fig. 6. Expanded arc furnace for vaporization of powders, (Whyman ${ }^{22}$ ).

This effect had earlier been observed by Maecker, ${ }^{24}$ who noted that where any current-carrying plasma column is constricted, there exists a radial component of current flow. The resulting magneto-hydrodymanic (MHD) forces give rise to a pressure drop in the region of the constriction. Thus there is a low pressure zone in the vicinity of the cathodic arc root. This gives rise to an inflow of gas, resulting in the well-known cathode jet in the conventional carbon arc. Maecker also observed that this natural convective flow permitted the entrainment into the arc column of carbon particles released in the vicinity of the arc root.

While efficient particle injection into a simple plasma column is difficult, the exploitation of these MHD forces can ensure efficient particle entrainment into the currentcarrying region of the arc. Thus the Maecker effect has been employed in a device described by Sheer et al. ${ }^{25,26}$ By maintaining an arc between a shielded tungsten cathode placed on the axis of the device, and three porous carbon transpiration-cooled anodes set around the axis above the cathode, an extended arc column is stabilised in space (Fig. 7). A powder-laden gas may be efficiently introduced into this arc column in the low pressure region which exists around the cathode tip. By feeding gas at high velocity into this zone the natural convection may be enhanced, and the introduced gas may be drawn into the column with up to $100 \%$ efficiency. ${ }^{25}$ If this gas feed is laden with particles, these particles are likewise drawn into the column. ${ }^{26}$ The effect appears to be self-enhancing; i.e. up to a certain point, increase in gas flow or powder feed rate results in heat removal in the cathode region and greater constriction of the arc. This in turn results in more efficient entrainment of the powder-laden gas stream.

This device, using the fluid convection cathode 


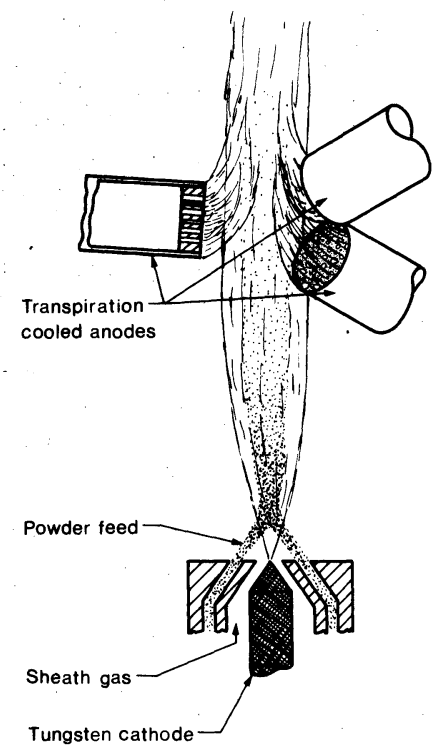

Fig. 7. Fluid-convection cathode, transpiration cooled anode device for particulate treatment. ${ }^{25}$

(FCC), has been used as a heater for reactive gases. ${ }^{25}$ Thus while the cathode itself must be shrouded in a gas inert to hot tungsten, the major gas flow, injected via an annular orifice around the cathode, may consist of virtually any gas (see Fig. 7). Again this outer gas flow may carry a powder stream, which may be heat treated or even substantially vaporized. ${ }^{26}$ Constriction of the arc in the cathode region leads to very high local gas temperatures, and temperatures of over $20,000^{\circ} \mathrm{K}$ have been observed. Such high temperatures lead to correspondingly high rates of heat transfer by conduction and radiation. The approach would appear to be very promising for heat treatment, chemical reaction, or partial vaporization of the feedstock. However for total vaporization it would appear to suffer some disadvantages relative to other approaches mentioned above.

It is interesting to note that the similarities between the FCC heater with 3 transpiration anodes and the Ionarc heater which uses a comparable shielded cathode and 3 consumable carbon anodes ${ }^{27}$ (Fig. 8). Powders are directed into the cathode jet region of the Ionarc heater by a series of ports set around the cathode orifice, and the Maecker effect will again be important in ensuring successful entrainment of particles into the currentcarrying region of the arc.

At the National Physical Laboratory a related device has been studied. ${ }^{28}$ Again, powders are injected into the Maecker pumping zone around a shielded cathode, but with our experience with transferred arcs between plasma jets we have chosen to employ 3 plasma jets as anodes. This assembly gives reliable operation with effectively non-consumable electrodes and permits the use of oxidising atmospheres when required. The equipment appears very promising for such applications as particulate spheroidization and reaction, and when operating at $120 \mathrm{~kW}$ it is possible to treat refractory powders at over $1 \mathrm{~kg} / \mathrm{min}$ to effect greater than $95 \%$ spheroidization.

Remarkably high loadings (e.g. $1 \mathrm{~kg}$ of $100-200 \mu \mathrm{m}$ particles fed to $801 / \mathrm{min}$ at STP of gas) may be achieved in this type of device. Higher loadings might be achieved

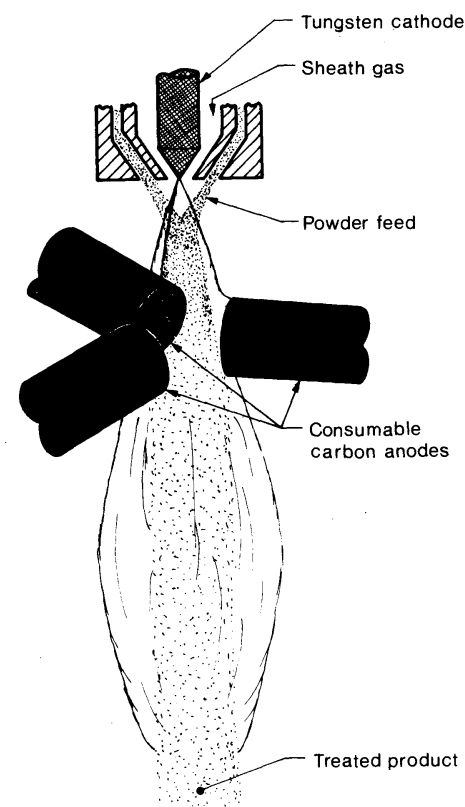

Fig. 8. Ion-arc heater for particulate treatment. ${ }^{27}$

in a fluidized bed, but at high temperatures the conventional fluidized bed may present problems of containment and agglomeration of molten particles. The use of a plasma-heated spouted bed as described by Bonet et al. ${ }^{29}$ permits a solution to some of these. Where such a spouted bed permits a comparable entrainment of particles, i.e. equally good contact between the particles and the current-carrying region of the arc, and where collection and containment problems are overcome, then the two approaches would appear capable of achieving comparable results.

It is because of the very multiplicity of devices which have been used to effect the simultaneous processes of heat and mass transfer that this paper has commenced with a description of the devices rather than with a review of the theoretical treatment of the processes occurring. If a device were to be selected for achieving the most efficient heat and mass transfer, then it might well be one of the transferred arc devices with FCC for a purely particulate approach, or a transferred arc centrifugal furnace if the material is to be treated as a liquid film. No theoretical treatment of either process has yet been published. Indeed there have been relatively few theoretical studies of simultaneous heat and mass transfer in plasma systems, and fewer still in which the theoretical predictions have been coupled with any attempt at experimental verification.

\section{THEORETICAL ASPECTS OF VAPORIZATION}

Rains and Kadlec reported the results of experiments in which alumina powders of various particle sizes were fed into an argon induction plasma at powers between 5 and $7 \mathrm{~kW}^{30}$ Vaporization of the particles (primarily as $\mathrm{Al}$, $\mathrm{Al}_{2} \mathrm{O}$ and $\mathrm{AlO}$ species) was observed, and after rapid quenching (by injection of hydrogen, carbon monoxide or methane into the plasma tail flame), it was possible to collect a pyrophoric powder containing a substantial percentage of free aluminium metal. The conversion was highest when quenching with methane, the presence of 
carbon minimizing the partial pressure of oxygen, and thus suppressing the back reaction to oxide. Any decomposition of methane on the hot alumina particles might also be expected to increase the conversion to metal by simple carbothermic reduction.

The degree of conversion was found experimentally to increase with decrease in powder feed rate or particle size, and with increase in plasma input power. By assuming that the percentage conversion corresponded to percentage vaporization, a qualitative agreement was achieved with estimates of the degree of vaporization obtained using a simple computer model for the process. In this model it was assumed that surface heat transfer was the rate-determining step, but no allowance was made for the heat absorbed by the diffusing vapours. By simultaneous solution of the heat, mass and momentum balance equations, estimates of the degree of vaporization showed similar trends to the experimentally determined percentage conversions, but were in error by $\sim 100 \%$. The disparities may be due, as suggested by Waldie, ${ }^{31}$ to the neglect of the heat absorbed by the diffusing vapours or possibly to the neglect of non-continuum effects. However since some degree of back reaction appears inevitable it may be unreasonable to expect that the degree of vaporization should correspond any more closely to the degree of conversion than is observed in practice.

The processes involved in the vaporization of oxides in an induction plasma have been considered in somewhat greater detail by Borgianni et al., ${ }^{32}$ who treated alumina and certain other oxides in argon plasmas at $3.5-5.5 \mathrm{~kW}$. The partially dissociated oxide particles were collected on a water-cooled cold finger and analysed. It was assumed that the quench rate was very much higher than the rate of back reaction and that the analysed product was representative of the composition of the plasma. The degree of decomposition of alumina was found to be unaffected by variation in mass flow from $8 \times 10^{-4}$ to $1.8 \times 10^{-2} \mathrm{~g} / \mathrm{sec}$, but the other trends were similar to those observed by Rains and Kadlec. The percentage of free aluminium was found to depend on the input power, particle size and distance of the quench from the injection point. A maximum of $12 \%$ free metal was achieved $(60 \mu \mathrm{m}$ particles, $5.5 \mathrm{~kW}$ input power) by collecting material at the end of the luminous region of the plasma.

In their theoretical treatment Borgianni et al. assumed that at any point in time, and for a particles of diameter $D$ at temperature $T_{p}$ in a gas of temperature $T_{g}$, the following heat balance applied:

$$
\begin{aligned}
\pi D^{2} h\left(T_{g}-T_{p}\right)+h_{r}\left(T_{g}\right) & =q_{p}\left(\mathrm{~d} T_{p} / \mathrm{d} t\right) \\
& +\Delta H_{T_{d}}(\mathrm{~d} n / \mathrm{d} t)+\pi D^{2} \epsilon_{p} \sigma T_{p}^{4}
\end{aligned}
$$

where $h$ is the heat transfer coefficient by the processes of conduction and $h_{r}\left(T_{g}\right)$ represents the contribution of radiation to the heat flux to the particle. Of the terms on the right hand side of the equation, $q_{p}\left(\mathrm{~d} T_{p} / \mathrm{d} t\right)$ represents the enthalpy gain of the particle $\left(q_{p}\right.$ being the heat capacity of the particle), $H_{T_{d}}$ is the enthalpy of decomposition and $\mathrm{d} n / \mathrm{d} t$ the rate of decomposition of the particles in mol/sec. The final term in which $\epsilon_{p}$ is the emissivity of the particle and $\sigma$ the Stefan-Boltzmann constant, represents the radiative heat loss of the particle. Borgianni et al. assumed that under the conditions applying the radiative gain in heat of the particle from the gas was small, and could be equated approximately to the radiative heat loss term. The two radiative terms were therefore ignored.

In calculating the heat transfer coefficient $h$, it was assumed that the relation

$$
\mathrm{Nu}=2.0+0.6(\operatorname{Re})^{1 / 2}(\operatorname{Pr})^{1 / 3}
$$

could be applied where the Nusselt number is given by $\mathrm{Nu}=h . \mathrm{D} / \chi(\chi=$ thermal conductivity of argon), the Reynolds number by $\mathrm{Re}=\mathrm{DV} \rho_{g} / \mu(\mathrm{V}=$ the velocity of gas relative to the particle; $\mu=$ the gas viscosity; $\rho_{g}=$ the gas density) and the Prandtl number by $\operatorname{Pr}=C_{p} \mu / \chi$ ( $C_{p}=$ the specific heat of particle). The overall heat transfer coefficient is then given by

$$
h=(\chi / D)\left[2.0+0.6(\mathrm{Re})^{1 / 2}(\mathrm{Pr})^{1 / 3}\right]
$$

The thermal conductivity $\chi$ was assumed to be the sum of three contributions

$$
\chi=\chi_{\text {atoms }}+\chi_{\text {ambipolar }}+\chi_{\text {electrons }}
$$

for which values were taken from the literature. It was necessary to use an average value for the boundary layer calculated using the relation

$$
\bar{\chi}=\int_{T_{p}}^{T_{g}} \chi \mathrm{d} T /\left(T_{g}-T_{p}\right)
$$

and the other quantities in the relations for $\mathrm{Re}$ and $\mathrm{Pr}$ were calculated at the mean temperature of the boundary layer.

The overall heat transfer coefficient $h$ calculated in this way makes no allowance for the enthalpy taken up by the mass counterflow of the evolved vapour. Above the decomposition temperature a modified value was therefore used by taking account of the overall enthalpy change from $T_{p}$ to $T_{g}$, and the enthalpy of decomposition at $T_{d \cdot}{ }^{33}$ Allowance was also made for the fact that the vaporizing species represent an additional heat sink within the boundary layer. ${ }^{34}$

The equation of motion was calculated assuming the applicability throughout of Stokes law, but allowing for a change in diameter in time (this increasing with temperature until the decomposition temperature $T_{d}$ and thereafter diminishing). For the velocity and viscosity of the plasma, the values were assumed to be those applying at the mean temperature at the boundary layer.

The heat taken up by particles was thus calculated as a function of time, and compared with results derived from chemical analysis of the quenched products. The results of such a comparison are shown for two particle diameters in Fig. 9, in which (in the absence of any direct measurements of plasma temperature) the dotted lines represent the calculation for isothermal plasma temperatures and the continuous line shows the results calculated assuming the particles were travelling down the axis through a temperature regime similar to that observed in a comparable argon plasma by Vurzel et al.$^{35}$ It appears that the smaller $(60 \mu \mathrm{m})$ particles pass through a lower temperature regime than the larger particles $(135 \mu \mathrm{m})$ and the authors suggested that this resulted from a greater radial spread of the smaller particles in passing through the plasma. This was attributed to the effects of thermophoresis which exist in regions of high temperature gradient $^{36,37}$ but may also result from the magnetohydrodynamic recirculating gas flows which exist in induction plasmas. ${ }^{3}$ 


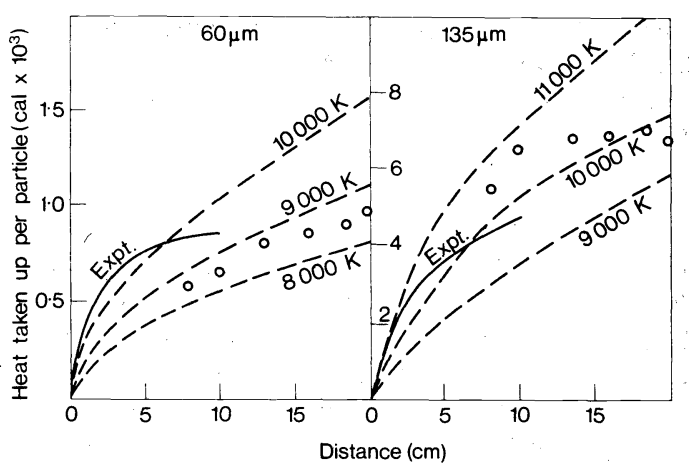

Fig. 9. Heat absorbed by $\mathrm{Al}_{2} \mathrm{O}_{3}$ particles (diameter $60 \mu \mathrm{m}$ and $135 \mu \mathrm{m})$, as a function of distance from the injection point. Continuous lines, curves calculated assuming the temperature profile along plasma axis given by Vurzel et al.;5 dotted lines, isothermal curves; circles, experimentally determined.

In view of the complexities and approximations involved, the extent of agreement between experimental and practical data is of considerable interest. The authors note that better agreement should be possible by making better allowance for the gas temperature regime through which the particles pass, by improvements in calculating the laws of motion of the particle, and by making better allowance for the effect of vapour species on the thermal conductivity of the boundary layer.

Full allowance for this last effect would be very difficult, but Capitelli et al. extended the above work by a comparable study in which alumina particles were injected into an argon induction plasma containing 5 or $8 \%$ of nitrogen. ${ }^{38}$ Their theoretical analysis, which assumed that the electron and gas temperatures of the boundary layer were equal, showed that in the presence of dissociated and ionising nitrogen species the heat transfer rates were markedly increased, the probable nonequilibrium nature of the plasma boundary layer being expected to increase the heat transfer rate still further. However attempts at experimental verification of these conclusions indicated a reduced level of decomposition, presumably as a consequence of the shorter length and the lower mean temperature of the argon-nitrogen plasmas.

Johnston $^{39}$ has also developed the calculation method of Borgianni et al. to take account of the radiative heat loss of particles, together with the radiative heat gain from neighbouring particles in the plasma flow. It was found that doubling the density of $250 \mu \mathrm{m}$ particles (from $1 \times 10^{7}-2 \times 10^{7} \mathrm{~m}^{-3}$ ) in an electrically augmented flame (assumed to be at a constant temperature of $4000^{\circ} \mathrm{K}$ ) led to a $10 \%$ reduction in decomposition time. At these gas temperatures the contribution of radiation from the gas was again assumed to be minimal.

In a very extensive theoretical study Bonet $e t$ al. have considered the processes involved in the evaporation of a spherical particle in somewhat greater detail. ${ }^{40}$ It was assumed that no reaction took place between the particle and the plasma gas, that the particle was large in comparison with the mean free path of the plasma species, and that the plasma was in local thermodynamic equilibrium. It was also assumed that the vapour species formed only a minor proportion of the total plasma. This latter assumption represents a rather serious limitation of the method, in that any efficient vaporization technique will be required in practice to yield a high partial pressure of the volatile species. However under the assumed conditions, Bonet et al. were able to derive equations for heat and mass transfer which applied under varying physical properties of the plasma. The relative importance of the various effects considered is set out in Table 1 . The treatment for the separate cases of optically thick and optically thin thermal plasmas, and the solution of the various equations involved by the method of perturbation, are given in the above paper.

The treatments mentioned above have assumed that the plasma was in a state of local thermodynamic equilibrium with all plasma species at the same effective temperature, and also that vapour species form a relatively small proportion of the plasma gas. The situation is, however, very different in all the devices listed above which offer high thermal efficiencies. In each of these, the processes of electrical heating of the gas and of transferring that heat to the particle are not separated, but are combined in one process.

This means that the plasma may be far from local thermodynamic equilibrium, and this very fact will promote a greatly enhanced rate of heat transfer. Thus in the Ionarc ${ }^{27}$ and fluid convection cathode devices, ${ }^{22,23,25,26,28}$ the particles are heated in the currentcarrying region of the arc in which, in the presence of a large proportion of cooler particles, the electron temperature will be substantially higher than the mean gas temperature, and the heat and mass transfer rates will be significantly improved. Again, at the high local temperatures observed in the fluid convection cathode device, ${ }^{25}$ the contribution of radiation from the gas will be considerable. In those devices where the material to be treated forms part of the electrode system, e.g. the high intensity arc, ${ }^{6}$ or the transferred arc centrifugal furnace, ${ }^{17}$ there also exists the added effect of the high density of

Table 1. Relative importance of various effects on vaporization of a spherical particle in a thermal plasma, Bonet $e t$ al. $^{40}$

\begin{tabular}{lccc}
\hline \multicolumn{1}{c}{ Phenomenon } & $\begin{array}{l}\text { Very fine } \\
\text { particles }\end{array}$ & Fine particles & Large particles \\
\hline $\begin{array}{l}\text { Radiation from particle } \\
\text { Thermal conductivity of gas }\end{array}$ & $\begin{array}{l}\text { Major } \\
\text { effects }\end{array}$ & $\begin{array}{c}\text { Major } \\
\text { effects }\end{array}$ & $\begin{array}{c}\text { All } \\
\text { effects } \\
\text { have } \\
\text { Thermal diffusion }\end{array}$ \\
$\begin{array}{l}\text { Fick diffusion } \\
\text { Radiation from plasma } \\
\text { Dufour effect } \ddagger\end{array}$ & Negligible & Minor & importance \\
Forced convection & & effects & \\
\hline
\end{tabular}

†Flow of matter caused by a temperature gradient.

$\ddagger$ Flow of heat resulting from a concentration gradient. 
energy release which exists in the anode fall zone, coupled with resistive heating of the substrate, and a full treatment of these subjects remains to be undertaken.

\section{CONDENSATION}

The subject of heat and mass transfer during vaporization in a thermal plasma has been considered separately from the second broad area to be discussed in this paper, that of condensation from a plasma-heated system. An all embracing theory would include both areas in the general case, but because the temperature regimes and experimental difficulties encountered in practice are very different, they are best considered as separate subjects. Again, the practical aspects will be discussed first.

Condensation from the plasma-heated vapour has two important practical applications: the deposition of condensate on a substrate (a process which may involve heterogeneous nucleation on the substrate), and the production of fine particles (homogeneous nucleation, or nucleation by ions present in the plasma). Growth by heterogeneous nucleation on a substrate is a particularly important area of technology in low pressure plasmas, but these are beyond the scope of the present paper. Such growth in thermal (atmospheric pressure) plasmas has received relatively little attention, but one of the first and major practical applications in the field is the growth of silica boules from the oxidation of silicon tetrachloride in an oxygen induction plasma. ${ }^{41}$ This relatively expensive route to silica is used only when the highest purity product is required. It was used first for the production of hydroxyl-free silica for spectroscopic purposes, where conventional boule growth in the oxy-hydrogen flame was unsatisfactory, but more recently, the method has also been employed for the production of pure and doped silicas for use in the manufacture of optical fibres. ${ }^{41,42}$

Silicon tetrachloride vapour is passed centrally through the oxygen plasma, and the stream of vapour products is directed on to the hot surface of the boule. It is probable that growth occurs as a mixture of both heterogeneous nucleation on the boule surface and homogeneous nucleation of fine silica particles, which then impact on the surface. Conditions are quite critical if the collection efficiency is to be high, and if the silica is to be deposited bubble-free in the correct viscous state. If the temperature is too low, growth occurs as a porous deposit (which can be sintered into a dense form in a separate process ${ }^{43}$ ), and if the temperature is too high, the silica may be too mobile and the boule will not be self-supporting. In addition, deposition efficiency will be reduced. Adjustment of aerodynamic flow patterns, temperature gradients, vapour pressure, and in particular the degree of supersaturation at the boule surface, are clearly critical, and the achievement of an efficient process appears to have evolved by considerable patient development rather than by resort to theoretical analysis.

A subject attracting widespread attention by workers in the field of thermal plasmas is the process of homogeneous condensation to produce fine particles in suspension in the hot gas stream. The method has been used to prepare ultrafine particles of oxides, and other refractory materials, and its use to prepare titania pigments, and thixotropic silica powders, represents two of the most promising applications of thermal plasmas to date.

A note of caution may be relevant at this point. The condensation processes to be discussed below occur in plasma-heated systems, however they do not in general take place at plasma temperatures. Their relevance to workers studying thermal plasmas is that plasmas are used to generate the required hot gas streams, or to provide the stream of vaporized material. However, it may prove desirable to effect the condensation reaction at much lower temperatures if the product is to be achieved in the required form, or if the specific energy requirement is to be minimized. In certain cases, for example the production of oxide powders, the process of interest has much in common with that occurring during a typical flame synthesis reaction.

The commercially important condensation of silica as an ultrafine powder has been extensively studied. Ultrafine silicas prepared by chemical routes are used on a scale of some tens of thousands of tonnes per annum for the control of viscosity of resins, varnishes, paints etc., as reinforcing fillers and in many other applications. These materials are generally made either by the vapour phase hydrolysis of silicon tetrachloride in an oxy-hydrogen flame, or by the supercritical hydrolysis of ethyl silicate ester. However, a number of groups have attempted to produce active silicas by direct vaporization of natural silica followed by condensation under suitable conditions. ${ }^{14,26,44-47}$ The vaporization aspects have been discussed above, but the condensation process will now be considered in greater detail.

The required condensation conditions are of course critically dependent on the nature of the desired product. While the precise structure of the sub-surface material in a thixotropically active silica gel may be uncertain, it is widely accepted that these materials may be considered as an agglomeration of chains of ultrafine particles of amorphous silica, each of which has a surface coverage of silanol (Si-OH) groups, there being three distinguishable types. ${ }^{45,48}$ These are, firstly, isolated silanol groups which are sufficiently spaced to be unable to interact with each other, secondly, similar groups with neighbouring silanol groups which are close enough to permit hydrogen bonding between the two, and thirdly, hydroxyl groups arising from water adsorbed at the silica surface.

The unusual properties of this highly hydroxylated surface are essential in the use of Aerosil silicas for viscosity control in liquids. Thus, when suspended in a polar liquid (e.g. water), the viscosity of the liquid is relatively little changed, and the surface hydroxyl groups are saturated by water molecules. However if a similar proportion of silica is added to a non-polar solvent, a gel structure is set up and the viscosity rises very markedly. This property is the basis for the application of these silicas in problems of flow control, and is interpreted as resulting from cross-linking of adjacent chains by hydrogen bonding to form a loose network throughout the liquid. The structure breaks down on agitation but re-forms once motion of the liquid ceases.

It is clear that for a thixotropically active silica product, a high degree of hydroxylation is required. Thus in any vapour phase route to such products one must ensure that there is an adequate partial pressure of water, or of hydroxylated polymeric silicon-containing precursors in the condensation zone. However this is not the only condition which must be satisfied.

On heating an Aerosil, progressive loss of water takes place. Initially, adsorbed water is evolved, and subsequently, by condensation of neighbouring silanol groups, siloxane linkages ( $\mathrm{Si}-\mathrm{O}-\mathrm{O}-\mathrm{Si}$ ) are formed. Up to $200^{\circ} \mathrm{C}$ the water loss is reversible, but thereafter an irreversible change takes place and above $1100^{\circ} \mathrm{C}$ no surface silanol groups remain. The material is no longer 
thixotropically active and cannot be reactivated by subsequent treatment with water. The clear implication of this fact is that the temperature at which condensation takes place must be adequately low (typically temperatures in silica synthesis flames are in the region of $\left.1800-2100^{\circ} \mathrm{K}^{49}\right)$, and furthermore the cooling of the products thereafter must be rapid if the combined water is not to be lost.

It is, in principle, possible to produce an active product by vaporizing silica in an oxidizing or inert atmosphere, and by subsequent rapid quenching of this vapour in an atmosphere containing water. However there are several reasons why vaporization in a reducing environment is preferable. In the presence of a reducing agent, for example carbon, silica may be caused to vaporize at a temperature between 1800 and $1900^{\circ} \mathrm{K}$, as opposed to $3070^{\circ} \mathrm{K}$, which is the approximate temperature at which the vapour pressure of silicon-containing species reaches one atmosphere in a neutral environment. ${ }^{50}$ Vaporization under reducing conditions thus leads to a lower energy requirement, can permit the use of a lower overall gas flow, and in addition leaves less heat to be removed in the condensation zone. The effective quench rate may therefore be considerably higher than would be possible using an inert or oxidizing atmosphere for vaporization.

While relatively little data have been published on the experimental conditions required for the production of a thixotropically active silica product for thermal plasma, ${ }^{14,44-47}$ the above generalizations are borne out by the experimental results, and it is clear that the conditions required to effect vaporization are very different from those required for the condensation of a useful ultrafine powder.

Taking as an example work on silica treatment using a centrifugal furnace ${ }^{13,14,51}$ there is no difficulty in containing silica near its boiling point, using for example a nitrogen plasma. Under these conditions the vaporization of silica takes place and on cooling, for example by injection of cold air, it is possible to produce an ultrafine silica product. An electron micrograph of a typical sample is shown in Fig. 10. The characteristic appearance of such a product is one of chains of spherical particles, and such a product, even at high surface area, does not exhibit the high thixotropic activity of materials made by vapour phase hydrolysis of silicon tetrachloride.

As discussed above, in order to prepare a thixotropic product from a plasma system a low temperature condensation is required, in an atmosphere containing an adequate partial pressure of hydroxylated species. Such conditions may be achieved using a reducing agent containing hydrogen; indeed hydrogen, methane and ammonia have all been used as gaseous reducing agents for vaporizing silica from the centrifugal furnace. ${ }^{14,51}$ On reoxidation each provides an atmosphere laden with water vapour, and if the flame temperature is adequately low, thixotropic products are readily produced. Carbon is also a valuable reducing agent, permitting rather more efficient vaporization, but its use leads to a water-free atmosphere containing carbon monoxide and silicon monoxide. If oxygen (or air) is used as the oxidant a high flame temperature is observed, and even in the presence of water vapour it may prove difficult to cool the vapours rapidly enough to obtain an active product. However, water vapour itself may be used as an oxidant, and in this case the carbon monoxide is not reoxidized. The flame temperature is thus much lower and there is no difficulty in obtaining a highly active product, which has both the appearance and properties of a conventional Aerosil. The electron microscope reveals a web-like structure, Fig. 11, similar to that of Aerosil, but very different from that of the inactive silica prepared in the absence of water (Fig. 10). Table 2 shows a comparison of the surface area and

Table 2. Comparison of properties of two types of plasmaproduced silica with those of a conventional product

\begin{tabular}{lccc}
\hline & $\begin{array}{c}\text { Surface area } \\
\left(\mathrm{m}^{2} \mathrm{~g}^{-1}\right)\end{array}$ & \multicolumn{2}{c}{ Viscosity $\begin{array}{c}\text { Speed 1 } \\
\text { Speed 2 }\end{array}$} \\
\hline Aerosil 200 & 202 & 122.6 & 124.1 \\
$\begin{array}{l}\text { Plasma produced silica } \\
\text { (a) Non-hydroxylated }\end{array}$ & 184 & 103.6 & 109.0 \\
(b) Highly hydroxylated & 209 & 125.5 & 126.4 \\
\hline
\end{tabular}

†Viscosities measured (with a Ferranti Viscometer) using a $1 \% \mathrm{w} / \mathrm{w}$ dispersion of silica in tritolyl phosphate at $20.8^{\circ} \mathrm{C}$. This liquid alone gave viscosities of 97.6 and $96.7 \mathrm{cP}$ at speeds 1 and 2 respectively.

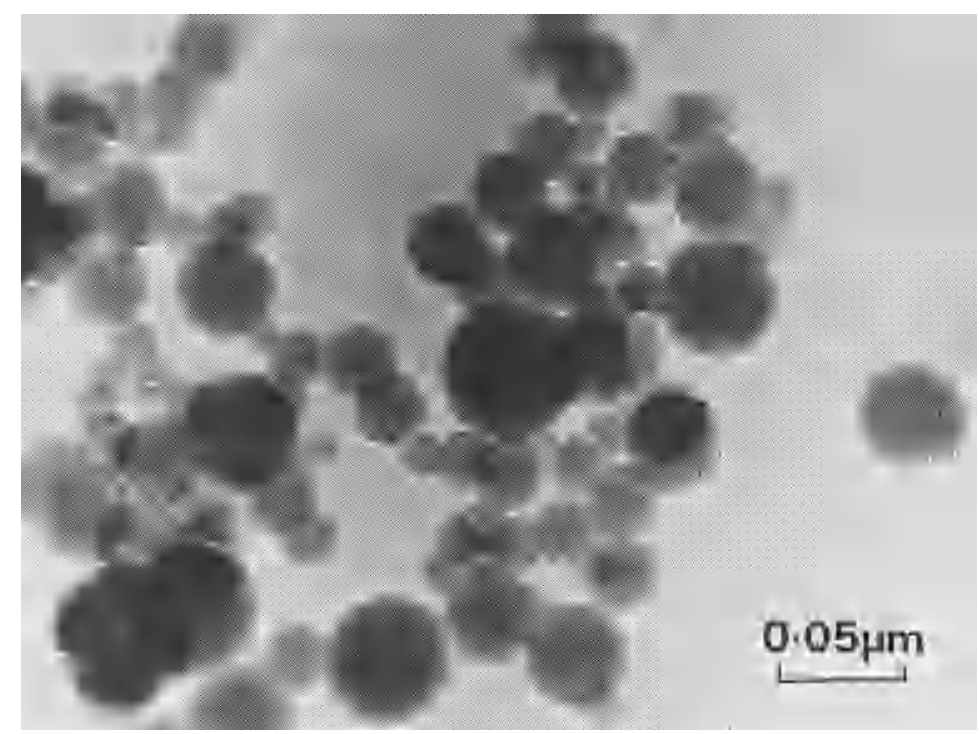

Fig. 10. Silica powder condensed from plasma under water-free conditions. ${ }^{14}$ 


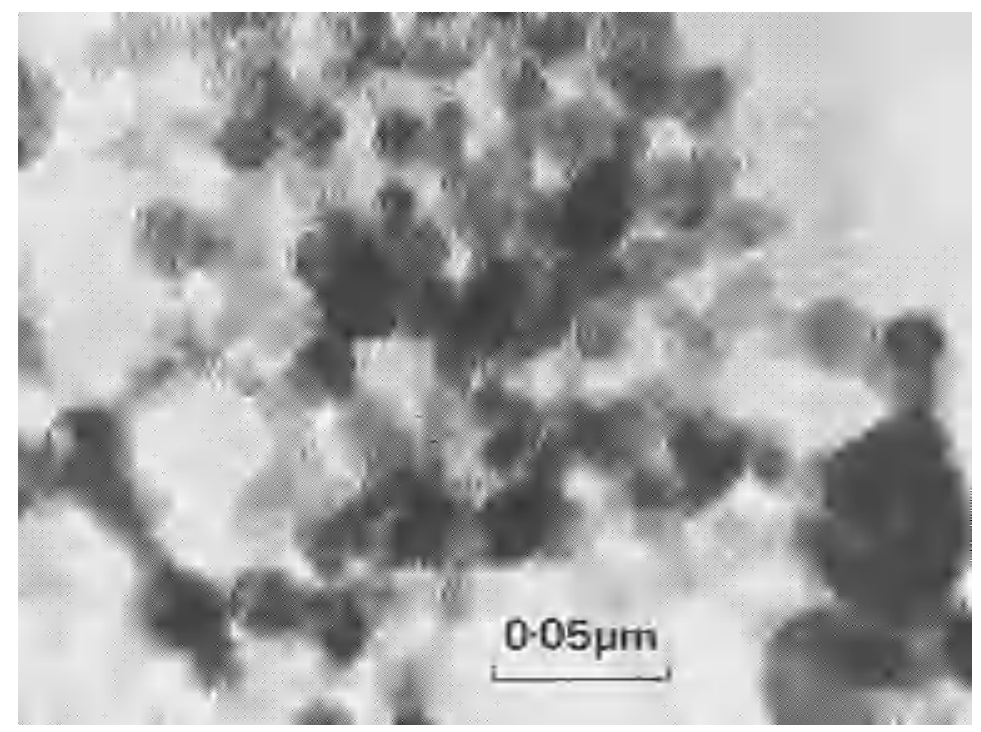

Fig. 11. Thixotropically active silica condensed from plasma in the presence of water vapour. ${ }^{14}$

viscosity characteristics of commercial Degussa Aerosil 200 and two silicas obtained by vaporizing a mixture of natural sand and petroleum coke in the centrifugal furnace. One of these was reoxidized in air, leading to a product of low hydroxylation, and the second was reoxidized by injection of steam to yield a product of high thickening capacity. ${ }^{51}$

It is apparent that this simple, single stage process yields a material comparable or better than those of the commercial product, the latter being obtained by a multistage process, which involves the complexities of containing and recycling hot chlorine-containing gas streams.

A comparable development has also been described by Illigen and Neugebauer, who have reported the vaporization of silicon monoxide from a conventional carbon arc furnace. ${ }^{45}$ On treating the vapour stream with steam or water sprays, an intermediate could be condensed, which on post-heating (for example at $500^{\circ} \mathrm{C}$ for $10 \mathrm{~min}$ ) could be converted to a thixotropic silica gel. Chemical and spectroscopic analysis of the intermediate indicated the presence of both silane and silanol groups, and suggested that this type of intermediate was formed by reactions of the form:

$$
\begin{aligned}
& \mathrm{SiO}(\mathrm{g})+\mathrm{H}_{2} \mathrm{O}(\mathrm{g}) \rightarrow \mathrm{SiO}_{2}+\mathrm{H}_{2}(\mathrm{~g}) \\
& \mathrm{SiO}(\mathrm{g})+\mathrm{H}_{2}(\mathrm{~g}) \rightarrow \mathrm{H}^{\mathrm{H}} \mathrm{Si}=\mathrm{O} \\
& \mathrm{SiO}(\mathrm{g})+\mathrm{H}_{2} \mathrm{O}(\mathrm{g}) \rightarrow \mathrm{H}-\mathrm{Si}^{\mathrm{O}}
\end{aligned}
$$

On heating in air or water vapour this intermediate loses hydrogen to form a gel with properties identical to those of a conventional Aerosil.

It is thus clear that if a thixotropically active product is to be prepared from a natural silica by a vaporizationcondensation route it is necessary to effect condensation in the presence of water vapour, either at a relatively low temperature, when a further treatment is required to yield the desired product, or at a higher temperature, when it is necessary to ensure that neither the flame temperature is too high nor the residence time too long, otherwise the silanol groups will lose water and the thixotropic activity of the product will suffer.

In passing, it may be noted that the condensation of reduced silica vapours in an inert atmosphere leads to a most unusual product, consisting predominantly of an ultrafine fibrous wool. Such a product has been obtained from the centrifugal furnace,,$^{13}$ and has also been observed by Kuhn in arc vaporized material. ${ }^{52}$ The whiskers appear to consist of disproportionated silicon monoxide, grown (at remarkable rates) by a vapour-liquid-solid mechanism, as observed in whisker growth of other materials. Kuhn reported that the spherical knob in which most of these whiskers terminate, consists predominantly of silicon. Despite the interesting morphology, which has yet to be observed in other plasma systems, no use has been found for the product, which is chemically unstable, and readily reoxidized to microcrystalline silica.

While silica and titania are the most important ultrafine oxides to be condensed from the plasma, some work has been done on the production of submicron aluminas. ${ }^{53,54}$ While there is not yet any comparable demand for this material, the process did serve as an interesting model system in a study of the condensation conditions. ${ }^{54} \mathrm{As}$ with silica, alumina may be caused to vaporize rapidly as reduced species. In the latter case carbon is the most satisfactory reducing agent, and $\mathrm{Al}$ and $\mathrm{Al}_{2} \mathrm{O}$ are the predominant aluminium-containing vapour species. Quenching the vapour in an argon atmosphere yields a pyrophoric ultrafine product containing much free aluminium metal, but reoxidation yields a sub-micron alumina powder. ${ }^{53}$ The condensation reaction was studied to establish what particle size and crystalline modifications of alumina could be prepared, and whether alumina could be prepared in a form analogous to a thixotropic silica gel. ${ }^{54}$

While some degree of control of particle size and properties may be achieved by varying the rate of flow of quench gas, this approach is not the most convenient, in that large volumes of gas may be required, and adequate mixing may be impossible to achieve on a microscopic scale. However, means must be found of controlling the temperature of the reoxidation zone, and here ther- 
modynamic calculations provide a useful predictive facility. It was estimated that, under typical experimental conditions, the temperature of the stream of reduced vapour within the furnace was approximately $2400^{\circ} \mathrm{K}$ and that the species contained in the vapour were in the following ratios: $\mathrm{Al}_{2} \mathrm{O}: \mathrm{C}: \mathrm{N}_{2}$ (plasma gas) =1:2:20. Using JANAF thermochemical data, ${ }^{55}$ the equilibrium concentrations of the various species were calculated, and these concentrations were then used in a further calculation in which the hypothetical adiabatic flame temperatures were computed assuming combustion in predetermined quantities (gross excess) of various potential oxidizing agents. The three selected were (per mole of alumina vaporized): $\mathrm{O}_{2}, 50 \mathrm{~mol}$ injected at $300^{\circ} \mathrm{K} ; \mathrm{H}_{2} \mathrm{O}$ (steam), $40 \mathrm{~mol}$ injected at $400^{\circ} \mathrm{K}$; and $\mathrm{CO}_{2}, 40 \mathrm{~mol}$ injected at $300^{\circ} \mathrm{K}$. The computed flame temperatures were then: $\mathrm{O}_{2} \cdot 1788^{\circ} \mathrm{K}, \mathrm{H}_{2} \mathrm{O} 1664^{\circ} \mathrm{K}$, and $\mathrm{CO}_{2} 1409^{\circ} \mathrm{K}$. The latter two oxidants gave lower temperatures than the oxygen flame temperature because the carbon monoxide present in the product gases is not reoxidized, and the heat of the combustion reaction is thus substantially less than that of the aluminium species in oxygen.

In this way it was established that, even at the high dilutions employed in practice, a considerable degree of control of flame temperature could be achieved by choice of oxidant. When the three oxidants were used experimentally, corresponding trends were observed in the crystalline structures and the surface areas of the three powders. Thus X-ray powder photography revealed that all contained $\gamma-\mathrm{Al}_{2} \mathrm{O}_{3}$, but the oxygen quenched material also contained $\delta-\mathrm{Al}_{2} \mathrm{O}_{3}$ a higher temperature modification. Furthermore there appeared to be an increasing proportion of amorphous alumina in the series $\mathrm{O}_{2}<\mathrm{H}_{2} \mathrm{O}<\mathrm{CO}_{2}$, corresponding to the decreasing flame temperature in the condensation zone. The surface areas of typical samples exhibited a corresponding trend, the powders quenched in oxygen, water and carbon dioxide yielding typical values of 72,123 and $137 \mathrm{~m}^{2} \mathrm{~g}^{-1}$ respectively.

The water-quenched powder was extensively studied in an endeavour to establish whether it was possible to prepare the alumina analogue of thixotropic silica. Materials were definitely prepared with properties closely comparable to those of commercially made fumed aluminas (prepared by flame hydrolysis of aluminium halides in an oxy-hydrogen flame ${ }^{56}$ ); however it appeared that the contained water was readily lost on heating and the morphology observed in high activity silicas was never demonstrated for an alumina product. If any similar materials are to be prepared by a plasma route, it would appear that the reoxidation will have to be effected at even lower temperatures than those employed above.

Ultrafine silicas, ${ }^{57}$ and aluminas ${ }^{58}$ have also been prepared by oxidation of the metal halides in the oxygen induction plasma. This device has also been used in the preparation of mixtures of oxides, and of mixed oxides by co-condensation. Barry et al. found that when chromia and titania were co-condensed from an oxygen plasma fed with the mixed metal halides there was extensive formation of the mixed oxide solid solution, ${ }^{59}$. but with chromia and alumina there was relatively little tendency to form such a solid solution. ${ }^{58}$ Presumably the difference in relative vapour pressures of the two oxides meant that condensation of chromia commenced only near the freezing point of the alumina droplets, which condensed first. ${ }^{60}$ Subsequently, by injecting reactants into the plasma tailflame, McPherson was able to raise the chromia vapour pressure substantially and to attain mixed oxides with up to $18 \mathrm{wt} \%$ of chromia in solution in the alumina phase. ${ }^{60}$

Mixed oxides have also been successfully prepared using the centrifugal furnace. Thus when alumina and magnesia are simultaneously evaporated from their $1: 1$ mixture, in the presence of carbon as reducing agent, the vapour may be reoxidised at the furnace exit to yield an ultrafine spinel $\left(\mathrm{MgAl}_{2} \mathrm{O}_{4}\right){ }^{61}$ Due to the differing volatilities of the two metal oxides it is difficult to control the relative proportions of the two components in batch vaporization experiments, but with continuous operation the relative proportions will (after the attainment of equilibrium) be identical to that found in the feed.

There have been numerous reports of the production of other ultrafine powders from plasma systems including carbides, ${ }^{62-64}$ nitrides $^{62,64-66}$ and metals. ${ }^{62,67,68}$ However in the studies reported there has been little attempt to control the quench conditions or the particle size of the product and these studies will not be considered further here.

Having discussed some of the factors which have been employed in practice to control the properties of fine powders condensed from the plasma heated system, some of the more theoretical treatments will now be considered. Again it may be noted that the vapour in the condensation zone will generally be well below "plasma" temperatures, and the treatments applied by workers in the fields of rocket combustion products and flame synthesis are thus highly relevant. Most early studies in the field assumed that nucleation and particle growth were the main mechanisms which controlled the ultimate particle size. A typical approach was that of Hermsen and Dunlap, in their treatment of the growth of alumina particles in an aluminium vapour flame. ${ }^{69}$

The nucleation of particles in metal vapour flames is generally accepted as a homogeneous process. In classical nucleation theory, it is assumed that in a supersaturated vapour, particles smaller than the critical nucleus are formed by collision of molecules of vapour with statistical probability, but being smaller than the critical nucleus, most re-evaporate. Only those which attain the size of the critical nucleus continue to grow. The radius of the critical nucleus $r_{c}$ is expressed by the Gibbs-Kelvin equation as

$$
r_{c}=\frac{2 \sigma N V_{b}}{R T \ln S}
$$

where $\sigma$ is the surface tension of the liquid, $N$ the Avogadro number, $V_{b}$ the volume per molecule of the substance condensing, $R$ is the gas constant, $T$ the absolute temperature, and $S$ the supersaturation. Applying this type of approach to the condensation of liquid alumina there are considerable uncertainties regarding the meaning of supersaturation (in a system in which the composition of the vapour differs chemically from that of the liquid), and, if the critical nucleus is very small, uncertainty regarding the value of the surface tension in relation to that of the bulk liquid.

However, noting these difficulties, Hermsen and Dunlap employed classical nucleation theory to calculate a nucleation rate, and thereafter assumed that particles grew by diffusion of condensing species to the surface, where they condense giving out a latent heat of condensation. ${ }^{69}$ Ignoring non-continuum effects, the rate of growth is then limited by the processes of transfer of heat from the condensing particle, and by the rate of 
diffusion of vapour species to the particle. Hermsen and Dunlap found that the latter limitation was negligible under the conditions assumed. They thus found a strong dependence of ultimate particle size upon the initial temperature of the system, with smaller particles resulting from lower initial temperatures. This effect is borne out by the experimental evidence. However they also found that as the partial pressure of reactants increased, so too did the nucleation rate, and in consequence the ultimate particle size decreased. Such effect is not always demonstrated in practice.

Among the factors noted by the above authors, but not included in the model, was the possibility of growth of particles by collision and coalescence. In a recent paper ${ }^{49}$ Ulrich showed that this process played a major part in determining the ultimate size of particles in silica synthesis flames, which involve the combustion of a premixed stream of silicon tetrachloride, hydrogen and air. Ulrich noted that the microscopic zones from which individual particles grow are very much smaller than the macroscopic turbulent eddies existing in the flame. This point is borne out by consideration of certain factors relevant to the production of a typical pyrogenic silica, see Table 3. It thus appears that growth may be considered to take place in a quasiquiescent fluid by the three microscopic processes viz., chemical reaction, nucleation, and Brownian motion.

Once chemical reaction has taken place to produce hypothetical $\mathrm{SiO}_{2}$ molecular vapour species, homogeneous nucleation will lead to condensation of the product. However, from calculations of critical nucleus size (using properties valid for bulk silica), Ulrich found that the critical nucleus might contain as few as one or two silica molecules, and that any larger particles would be stable under the conditions existing in the flame. Ulrich therefore concluded that the final particle size distribution in this system is not determined by chemical reaction (which is rapid), nor by nucleation, but rather by Brownian coagulation of the multiplicity of particles containing (initially) very few molecules of silica. Particles collide, and with a certain (unknown) sticking coefficient mutually adhere. Above the melting point of the oxide such particles coalesce. When the droplet has cooled to below the melting point coalescence no longer takes place, but rather interparticulate sintering occurs to produce the well-known chain-like aggregates. As Sutherland has shown, ${ }^{70}$ the morphology of the chains produced suggests that they are built up via accretion of small clusters of particles, rather than by addition of discrete particles. Further growth of aggregates ceases when the temperature drops below the sintering temperature of the material, and inter-particulate collisions become elastic.

The surface area of the product is determined by the size of the ultimate particle, which is in turn determined by the number of collisions which take place between molecular clusters up to the point at which the particle solidifies. Coagulation phenomena in systems where the Knudsen number (the ratio of molecular mean free path to particle diameter) is greater than 10 , may be calculated directly from kinetic theory, and thus Ulrich calculated, for example, product surface area as a function of residence time for two different initial particle size distributions (Fig. 12). Clearly the effect of initial distribution is soon dominated by Brownian growth, and the surface area of the eventual silica is thus unaffected by the early history of the reaction.

The rate of particle growth by chemical reaction was found to be strongly dependent on the initial concentration, but insensitive to flame temperature. However the ultimate size of the particle is dependent upon flame temperature, because growth by collision and coalescence of particles soon becomes predominant, and continues until the system has cooled to below the melting point of the oxide. It follows that the ultimate particle size, and surface area of the product, depends on the retention time at temperatures above the "minimum coalescence temperature", and in a conventional flame this retention time increases with increasing initial flame temperature.

Ulrich found that available experimental data on both oxide and carbon-forming (i.e. soot-forming) flames were consistent with the collision-coalescence model, but generally a quantitative comparison was difficult because of uncertainties in the residence times for particle growth in these flames. In carbon-forming flames growth of liquid

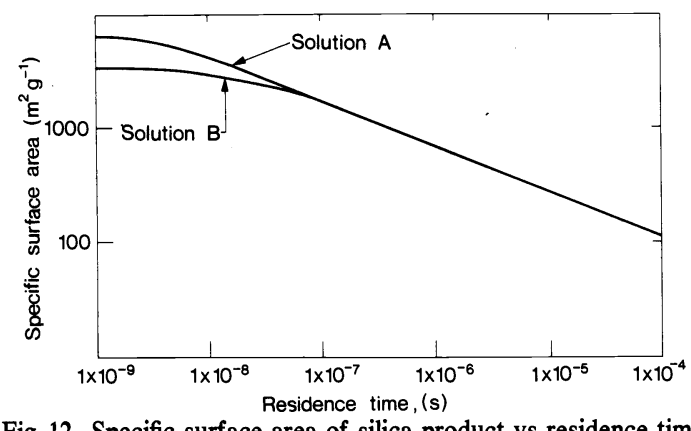

Fig. 12. Specific surface area of silica product vs residence time computed by Ulrich. ${ }^{49}$ Conditions; temperature $2000^{\circ} \mathrm{K}$, pressure $1 \mathrm{~atm}$, silica mole fraction 0.135 , sticking coefficient unity Solution A assumes initial population of silica particles all $4 \AA$ diameter, solution B, equal numbers of $4 \AA$ and $8.6 \AA$ particles.

Table 3. Characteristic dimensions of the particle environment in silica synthesis flames (Ulrich ${ }^{49}$ )

\begin{tabular}{|c|c|c|}
\hline & $\begin{array}{l}\text { Standard } \\
\text { silica }\end{array}$ & $\begin{array}{l}\text { High surface } \\
\text { area silica }\end{array}$ \\
\hline Surface area & $200 \mathrm{~m}^{2} \mathrm{~g}^{-1}$ & $400 \mathrm{~m}^{2} \mathrm{~g}^{-1}$ \\
\hline Equivalent particle diameter & $136 \AA$ & $68 \AA$ \\
\hline $\begin{array}{l}\text { Diameter of spherical fluid volume element } \\
\text { required to produce an average particle }\end{array}$ & $5440 \AA$ & $2720 \AA$ \\
\hline $\begin{array}{l}\text { Ratio of fluid element diameter to } \\
\text { particle diameter }\end{array}$ & 40 & 40 \\
\hline Number of $\mathrm{SiO}_{2}$ molecules per particle & $2.9 \times 10^{4}$ & $3.64 \times 10^{3}$ \\
\hline $\begin{array}{l}\text { Mean free path of typical gas molecules } \\
\text { at } 1 \mathrm{~atm} \text { pressure, } 2000^{\circ} \mathrm{K}\end{array}$ & $4800 \AA$ & $4800 \AA$ \\
\hline Minimum Knudsen number & 35 & 70 \\
\hline
\end{tabular}


high molecular weight hydrocarbon droplets again occurs by Brownian collisions, until, with loss of hydrogen, these droplets solidify and no longer coalesce..$^{71}$

A rather similar model to that discussed above has been used by Cozzi and Cadorin to predict the particle size distribution of titania particles obtained by oxidation of titanium tetrachloride in a lean carbon-monoxide-air flame. ${ }^{72}$ The major difference lies in the requirement with this system particles be produced of larger ultimate particle size (in the region of $0.2 \mu \mathrm{m}$ ), and in the assumption in the model that particle growth via surface reaction and condensation was significant over the major part of the residence time. In Ulrich's work these processes were assumed to be complete within a very small proportion of the total growth time. Cozzi and Cadorin's model leads to plots of the form shown in Fig. 13, in which the effect of the varying constants in the system may be observed. In the example shown nucleation is complete after some $5 \times 10^{-3} \mathrm{sec}$, and thereafter the particles grow by surface reaction and coagulation, the latter being more significant in the later stages of the process.

Recently Lillicrap and Lawton ${ }^{46}$ have developed the model of Ulrich for the condensation of submicron silica fumes, which were obtained by air-quenching a stream of vapour which had in its turn been formed by heating silica in a neutral atmosphere in a plasma furnace. Experimentally the surface area of the product was found to be approximately proportional to the volume of quench air. Agreement between the experimental results and theory was however not good, a possible reason for the divergence of results being the difficulty of maintaining accurate and reproducible values of such variables as the rate of
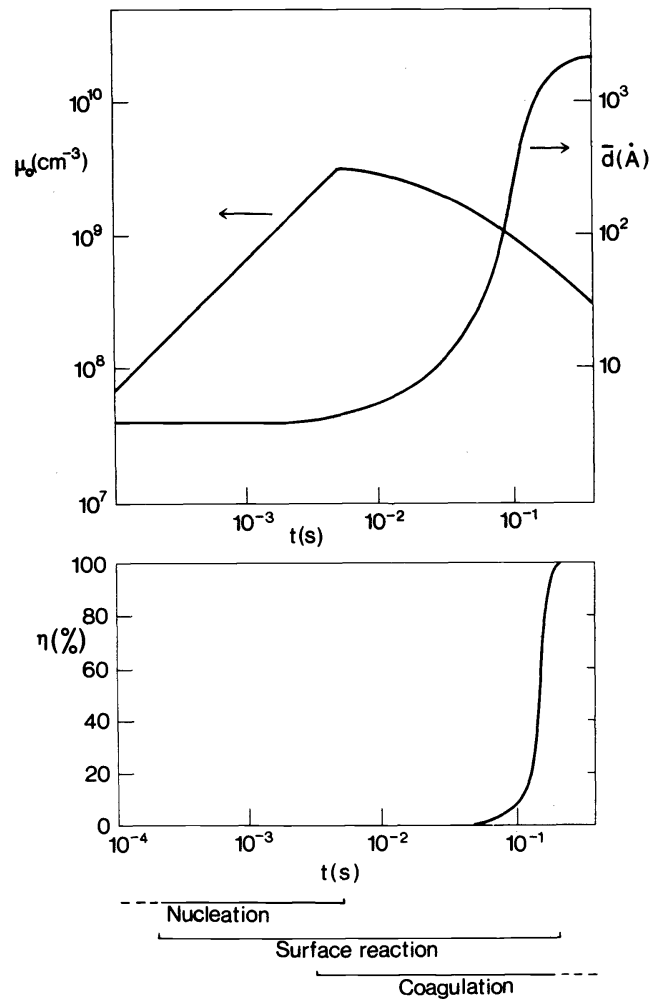

Fig. 13. Condensation of titania as computed by Cozzi and Cadorin, ${ }^{72}$ showing particle concentration $\left(\mu_{0}\right)$, mean particle diameter $(\overline{\mathrm{d}})$, and degree of conversion $(\eta)$ as functions of time. Nucleation rate $7 \times 10^{11} \mathrm{~cm}^{-3} \mathrm{sec}^{-1}$, surface reaction constant $1 \times 10^{-15} \mathrm{~cm}^{3} \mathrm{sec}^{-1}$, coagulation constant $7 \times 10^{-9} \mathrm{~cm}^{3} \mathrm{sec}^{-1}$, initial molecular concentration of $\mathrm{TiCl}_{4} 1.1 \times 10^{17} \mathrm{~cm}^{-3}$. production and enthalpy of silica vapour emerging from the plasma furnace. ${ }^{46}$

At this stage it may be relevant to sound a note of caution regarding all these theoretical treatments of oxide particle growth, particularly where they are applied to systems where the purpose of the experiment is the production of thixotropically active silicas. The theoretical treatments necessarily assume an oversimplified view of the reaction/condensation process and, as indicated above, there is strong evidence to suggest that the condensation of a thixotropically active product is in reality a very complex process. In practice the vapour will contain $\mathrm{SiO}$ together with $\mathrm{Si}_{2} \mathrm{O}_{2}, \mathrm{Si}_{3} \mathrm{O}_{3}$ and polymeric species. Reaction with water vapour will lead to species such as<smiles>O=[SiH](O)O</smiles>

and<smiles>[SiH3]O[GeH3]</smiles>

and no doubt complex polymeric species containing silane and silanol groups. Further heating of these polymeric hydroxylated species will cause evolution of water and hydrogen, and the thixotropic product will then be formed as discrete particles which thereafter aggregate, coalesce and later sinter into the well known grape-like clusters. No theoretical model yet postulated takes account of any such complex, multistage process.

In concluding this brief and selective survey, it appears that in each of the fields discussed above viz., vaporization, reaction and condensation, there are many promising areas for experimental study, including a number of fields of considerable commercial interest. However, for the present, our theoretical understanding and predictive capabilities leave considerable scope for improvement, particularly where they concern systems of real practical interest. It seems probable that the process of bringing together the practical and theoretical aspects of the subject will provide a challenge to both experimentalist and theoretician alike for some years to come.

\section{REFERENCES}

${ }^{1}$ I. G. Sayce, Advances in Extractive Metallurgy and Refining (editor M. J. Jones) p. 241. Inst. Min. Metal., London (1972).

${ }^{2}$ F. H. Howie and I. G. Sayce, Trans. Inst. Min. Metal. (Sect. C) 84, 231 (1975).

${ }^{3}$ J. D. Chase, J. Appl. Phys. 40, 318 (1969).

${ }^{4}$ B. Waldie, Trans. Inst. Chem. Eng. 49, 114 (1971).

${ }^{5}$ G. A. Farnell and B. Waldie, J. Phys. E, Sci. Instr. 6, 137 (1973).

${ }^{6} \mathrm{C}$. Sheer and S. Korman, Arcs in Inert Atmospheres and Vacuum, p. 169. Wiley, New York (1956).

${ }^{7}$ S. Korman and C. Sheer, Electrochem. Soc. Symp. Philadelphia (1966).

${ }^{8}$ M. H. D. Research Inc., U.K. Patent 1,205,576 (1970).

${ }^{9}$ W. R. Barnes, U.K. Patent 1,211,702 (1970).

${ }^{10}$ B. Gross, B. Grycz and K. Miklossy, Plasma Technology. Iliffe, London (1968).

${ }^{11}$ A. V. Grosse, P. J. McGonigal, W. S. Murphy and C. S. Stokes, Mat. Res. Std. 5, 173 (1965).

${ }^{12}$ B. Selton and J. A. Sheward, J. Mater. Sci. 4, 302 (1969).

${ }^{13}$ I. G. Sayce and B. Selton, Special Ceramics 5 (editor P. Popper), British Ceramic Research Association, Stoke on Trent, p. 157 (1972).

${ }^{14}$ D. A. Everest, I. G. Sayce and B. Selton, Symposium on 
Electrochemical Engineering (editor J. D. Thornton), Vol. 2, p. 2, 108, Institution of Chemical Engineers, London (1973).

${ }^{15}$ M. Foex, D. Yerouchalmi and R. Delmas, Compt. rend. 270(c), 1082 (1970).

${ }^{16}$ E. L. Bush and H. F. Sterling, U.K. Patent 1,313,467 (1973).

${ }^{17} \mathrm{~F}$. H. Howie and I. G. Sayce, unpublished work.

${ }^{18} \mathrm{H}$. Biegler, W. Neugebauer and H. Kempers, U.S. Patent $3,423,184$ (1969).

${ }^{19}$ F. H. Howie and I. G. Sayce, Rev. Int. Hautes Temp. Réfract. 11, 169 (1974).

${ }^{20}$ T. R. Shelley and J. A. Charles, Trans. Inst. Min. Metal. (Sect. C) 79, 259 (1970).

${ }^{21}$ T. R. Shelley and J. A. Charles, Trans. Inst. Min. Metal. (Sect. C) 83, 18 (1974).

${ }^{22}$ D. Whyman, J. Sci. Instr. 44, 525 (1967).

${ }^{23}$ J. W. Bryant, J. M. Cox and D. Whyman, J. Phys. E, Sci. Instr. 2 , 779 (1969)

${ }^{24}$ H. Maecker, Zeit. Phys. 141, 198 (1955).

${ }^{25} \mathrm{C}$. Sheer, S. Korman and S. F. Kang, Investigation of convective arcs for the simulation of re-entry aerodynamic heating, AFOSR-TR-74-1505, Contract F-44 620-69-C-0104 (1974).

${ }^{26} \mathrm{C}$. Sheer, S. Korman, D. J. Angier and R. P. Cahn, Fine Particles (editor W. E. Kuhn) p. 133. Electrochemical Society, Princeton, NJ (1974).

${ }^{27}$ Humphreys Corporation, U.K. Patents $1,317,918$ and 1,317,919 (1973).

${ }^{28}$ R. K. Bayliss, J. W. Bryant and I. G. Sayce, To be published.

${ }^{29}$ C. Bonet, G. Vallbona and M. Foex, Bull. Soc. Fr. Ceram. 94, 27 (1972).

${ }^{30}$ R. K. Rains and R. H. Kadlec, Metal. Trans. 1, 1501 (1970).

${ }^{31}$ B. Waldie, Chem. Eng. (Lon.) 261, 188 (1972).

${ }^{32}$ C. Borgianni, M. Capitelli, F. Cramarossa, L. Triolo and E. Molinari, Combust. Flame, 13, 181 (1969).

${ }^{33} \mathrm{~J}$. L. Engelke, Heat transfer to particles in the plasma flame, Report Stanford Research Institute, Menlo Park, California (1962).

${ }^{34} \mathrm{C}$. O. Bennett and J. E. Meyers, Momentum, Heat and Mass Transfer. McGraw-Hill, New York (1962).

${ }^{35}$ F. B. Vurzel, N. N. Dolgopolov, A. I. Maximov, L. S. Polak and V. I. Fridman, Kinetika i Termodinamika Khimicheski Reakskii v Nisko-Temperaturnoi Plasme (editor L. S. Polak) p. 223. Nauka, Moscow (1965).

${ }^{36}$ P. Epstein, Zeit Phys. 54, 537 (1929).

${ }^{37} \mathrm{~J}$.-P. Dauvergne and P. Le Goff, Rev. Int. Hautes Temp. Réfract. 4, 163 (1967).

${ }^{38} \mathrm{M}$. Capitelli, F. Cramarossa, L. Triolo and E. Molinari, Combust. Flame 15, 23 (1970).

${ }^{39}$ P. D. Johnston, Combust. Flame 18, 373 (1972).

${ }^{40} \mathrm{C}$. Bonet, M. Daguenet and P. Dumargue, Int. J. Heat Mass Transfer 17, 643 (1974).

${ }^{41}$ K. Nassau, T. C. Rich and J. W. Shiever, Appl. Optics 13, 744 (1974).
${ }^{42}$ J. A. Winterburn, Ger. Offen. 2,239,249 (1973).

${ }^{43}$ J. S. Flamenbaum, P. C. Schultz and F. W. Voorhoes, U.S. Patent 3,806,570 (1974).

${ }^{44}$ W. R. Barnes and D. Barby, U.K. Patent 1,211,703 (1970).

${ }^{45}$ A. Illigen and W. Neugebauer, U.S. Patent 3,674,430 (1972).

${ }^{46}$ D. C. Lillicrap and J. Lawton, I. Chem. E, Symposium Series No. 43, 24-1 (1975).

${ }^{47}$ S. Korman, C. Sheer, D. J. Angier and H. Shaw, Fine Particles (editor W. E. Kuhn) p. 153. Electrochemical Society, Princeton, NJ (1974).

${ }^{48}$ R. Bode, H. Ferch and H. Fratzscher, Kautschuk und Gummi Kunststoffe 20, 578, 699 (1967).

${ }^{49}$ G. D. Ulrich, Combust. Sci. Tech. 4, 47 (1971).

${ }^{50}$ H. L. Schick, Chem. Rev. 60, 331 (1960).

${ }^{51}$ I. G. Sayce and B. Selton, unpublished work.

${ }^{52}$ W. E. Kuhn, Ultrafine Particles (editor W. E. Kuhn), p. 156. Wiley, New York (1963).

${ }^{53}$ D. A. Everest, I. G. Sayce and B. Selton, J. Mater Sci. 6, 218 (1971).

${ }^{54}$ M. F. Barrett, D. C. Havard, I. G. Sayce, B. Selton and R. Wilson, Faraday Symp. 8, 149 (1973).

${ }^{55}$ JANAF Thermochemical Tables, US Departiment of Commerce, National Bureau of Standards, Washington, 2nd Edn (1971).

${ }^{56}$ Cabot Corporation, Technical Literature (1968).

${ }^{57}$ A. Audsley and R. K. Bayliss, J. Appl. Chem. 19, 33 (1969)

${ }^{58}$ T. I. Barry, R. K. Bayliss and L. A. Lay, J. Mater. Sci. 3, 229 (1968).

${ }^{59}$ T. I. Barry, R. K. Bayliss and L. A. Lay, J. Mater. Sci. 3, 239 (1968).

${ }^{60}$ R. McPherson, J. Mater. Sci. 8, 859 (1973).

${ }^{61}$ I. G. Sayce and D. A. Everest, U.K. Patent 1,369,585 (1974).

${ }^{62}$ E. Neuenschwander, J. Less Common Metals 11, 365 (1966).

${ }^{63}$ R. M. Salinger, Ind. Eng. Chem. Prod. Res. Develop. 11, 230 (1972).

${ }^{64}$ S. F. Exell, R. Roggen, J. Gillot and B. Lux, Fine Particles (editor W. E. Kuhn) p. 165. Electrochemical Society, Princeton, NJ (1974).

${ }^{65}$ H. Harnisch, G. Heymer and E. Schallus, Chem. Ing. Tech. 35, 7 (1963).

${ }^{66} \mathrm{H}$. Winterhager and K. Hanusch, Ber. dtsch keram Ges, 46, 181 (1969).

${ }^{67}$ CIBA Ltd, U.K. Patents $1,141,775$ and 1,141,776 (1969)

${ }^{68}$ S. M. L. Hamblyn, B. G. Reuben and R. Thompson, Special Ceramics 5 (editor P. Popper) p. 147. British Ceramic Research Association, Stoke on Trent (1972).

${ }^{69} \mathrm{R}$. W. Hermsen and R. Dunlap, Combust. Flame, 13, 253 (1969).

${ }^{70}$ D. N. Sutherland, Nature, 226, 1241 (1970).

${ }^{71}$ G. D. Ulrich, Comments, Twelfth Int. Symp. Combustion. p. 884 The Combustion Institute (1969).

${ }^{72}$ C. Cozzi and D. Cadorin, Combust. Sci. Tech. 5, 213 (1972). 